Comparing a discrete and continuum model of the intestinal crypt

This article has been downloaded from IOPscience. Please scroll down to see the full text article.

2011 Phys. Biol. 8026011

(http://iopscience.iop.org/1478-3975/8/2/026011)

View the table of contents for this issue, or go to the journal homepage for more

Download details:

IP Address: 129.67.187.35

The article was downloaded on 18/03/2011 at 19:10

Please note that terms and conditions apply. 


\title{
Comparing a discrete and continuum model of the intestinal crypt
}

\author{
Philip J Murray ${ }^{1}$, Alex Walter ${ }^{2}$, Alexander G Fletcher ${ }^{1,5}$, \\ Carina M Edwards ${ }^{3}$, Marcus J Tindall ${ }^{4}$ and Philip K Maini ${ }^{1,5}$ \\ ${ }^{1}$ Centre for Mathematical Biology, Mathematical Institute, 24-29 St Giles', Oxford OX1 3LB, UK \\ ${ }^{2}$ Oxford Centre for Collaborative Applied Mathematics (OCCAM), Mathematical Institute, 24-29 St \\ Giles', Oxford OX1 3LB, UK \\ ${ }^{3}$ Center for Modeling and Simulation in the Biosciences, Im Neuenheimer Feld 267, University of \\ Heidelberg, 69120 Heidelberg, Germany \\ ${ }^{4}$ School of Biological Sciences and Department of Mathematics and Statistics, University of Reading, \\ Whiteknights, Reading, Berkshire RG6 6AJ, UK \\ ${ }^{5}$ Oxford Centre for Integrative Systems Biology, Department of Biochemistry, South Parks Road, \\ Oxford OX1 3QU, UK
}

Received 16 September 2010

Accepted for publication 3 February 2011

Published 16 March 2011

Online at stacks.iop.org/PhysBio/8/026011

\begin{abstract}
The integration of processes at different scales is a key problem in the modelling of cell populations. Owing to increased computational resources and the accumulation of data at the cellular and subcellular scales, the use of discrete, cell-level models, which are typically solved using numerical simulations, has become prominent. One of the merits of this approach is that important biological factors, such as cell heterogeneity and noise, can be easily incorporated. However, it can be difficult to efficiently draw generalizations from the simulation results, as, often, many simulation runs are required to investigate model behaviour in typically large parameter spaces. In some cases, discrete cell-level models can be coarse-grained, yielding continuum models whose analysis can lead to the development of insight into the underlying simulations. In this paper we apply such an approach to the case of a discrete model of cell dynamics in the intestinal crypt. An analysis of the resulting continuum model demonstrates that there is a limited region of parameter space within which steady-state (and hence biologically realistic) solutions exist. Continuum model predictions show good agreement with corresponding results from the underlying simulations and experimental data taken from murine intestinal crypts.
\end{abstract}

\section{Introduction}

A healthy colonic tract is composed of several distinct layers of tissue and has a luminal surface that consists of regularly positioned, test-tube-shaped invaginations called crypts (see figure 1). Its outermost monolayer of epithelial cells, which acts as a protective barrier and absorbs nutrients, is attached to a basal lamina, which itself lies on top of a further tissue layer, the lamina propria.

There is a well-defined organizational structure within a homeostatic crypt: proliferative stem cells, which are believed to reside near the bottom of the crypt [32], divide to produce semi-differentiated transit cells that move up the crypt wall towards the luminal surface. Transit cells divide a maximum of four or five times before becoming further differentiated and non-proliferative. Upon reaching the top, the fully differentiated cells either undergo apoptosis and/or are shed into the lumen and transported away [11, 27]. The journey time of a cell from bottom to top of a murine colorectal crypt is thought to be between 2 and 3 days [28].

Molecular gradients confer positional information to cells, which is processed by subcellular molecular networks and influences cell behaviour. For example, canonical Wnt signalling has been identified as being an important regulator of transcriptional activity along the crypt axis (e.g. [10, 33]). Wnt molecules, whose distribution is graded along the crypt axis [13], bind at the cell surface, releasing transcription factors which, subsequently, migrate to the nucleus and initiate gene 


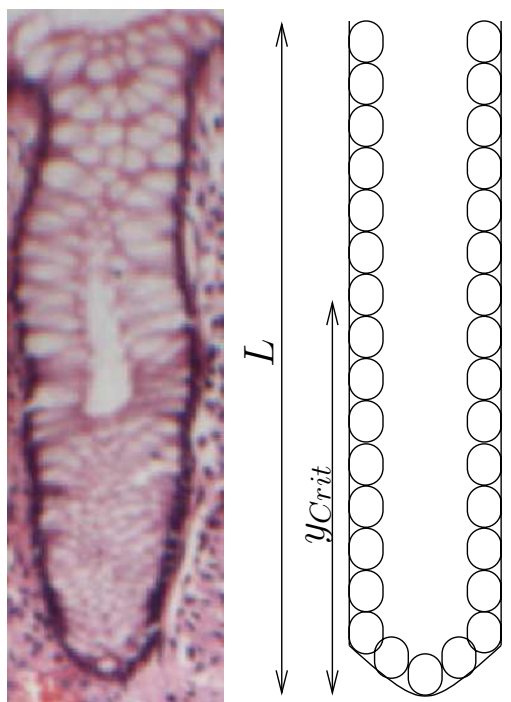

Figure 1. A section through a normal healthy crypt. Image supplied by kind permission of Trevor Graham, CRUK. A schematic illustration of the crypt continuum model. The parameters $L$ and $y_{\text {Crit }}$ denote the crypt height and the critical value of $y$ below which cells proliferate, respectively.

transcription. One such transcription factor, $\beta$-catenin, is known to influence cell proliferation, apoptosis, differentiation and migration [10]. Mutations that alter the balance between cell proliferation, differentiation and apoptosis within the crypt can lead to the formation of neoplasia [14, 22, 34]. For example, $85 \%$ of colorectal tumours have a mutation in the APC gene, a key regulator of Wnt pathway activity [1]. Hence, an understanding of the homeostatic regulation of normal crypts is essential for the understanding of the initiation and progression of diseases such as colorectal cancer.

Computational models have been used extensively in the study of crypt homeostasis [36]. Compartment models represent one of the more straightforward mathematical approaches: the populations of different cell types (e.g. stem, transit and differentiated) are modelled using ordinary differential equations (ODEs) [2, 16]. These models can be used to test hypotheses regarding the proliferative and differentiative behaviour of the various collective populations. However, ODE models cannot explicitly account for spatial regulation of cell behaviour, such as control by molecular gradients, or mechanical properties, such as cell adhesion and movement.

In a cellular automaton (CA) framework, discrete subpopulations of cells are tracked on a periodic lattice representing the crypt wall, with the automaton rules based upon available biological data, such as cell labelling studies and cell proliferation/movement rates $[17,18]$. These models have been used to validate verbal models of cell dynamics within the crypt and to place theoretical bounds on quantities such as the number of stem cells in the crypt. Drawbacks with the CA approach include: (a) the presence of lattice artefacts arising from the discrete nature of the cellular automaton; and (b) difficulty relating automaton rules to biophysical principles and measurements. These issues are avoided by the use of off-lattice models, where cell movement is continuous and occurs as a result of the consideration of an explicit definition of biomechanical forces. For example, Meineke et al [21] have considered an off-lattice model in which cells interact via a linear force law, with populations of stem, transit and differentiated cells tracked as they move, divide and differentiate. Van Leeuwen et al have extended the Meineke et al [21] framework by including Wnt-dependent regulation of cell proliferation and movement, thus coupling phenomena occurring at the subcellular, cellular and tissue scales. At the subcellular level, deterministic ODEs characterize molecular networks, such as cell-cycle control and Wnt signalling. The output of these subcellular models then determines the behaviour of each epithelial cell in response to intra-, interand extra-cellular cues.

While the Meineke et al [21] and Van Leeuwen et al [37] models provide a computational method for including increasing amounts of 'bottom-up' biological data, parameter space searches involving a vast number of simulations are required in order to develop insights into model behaviour at the cell population scale. For larger parameter spaces, this process becomes increasingly inefficient. For example, it is not clear a priori how the crypt simulations will perform if the crypt length is varied in order to apply the model to other regions of the intestine or species. These problems can be circumvented, to a certain extent, by considering continuum approximations to underlying discrete simulations [7, 19, 2326, 38]. Analysis and simulations of the continuum models can help to develop insight regarding governing features of the underlying simulations, and hence the consequences of the underlying biological assumptions upon which the models are based.

In this paper we investigate how the key processes of cell proliferation and movement are related in the intestinal crypt by developing a continuum model based upon the Meineke et al [21] and Van Leeuwen et al [37] simulation frameworks. The layout is as follows: in section 2 we consider a continuum approximation to the Meineke et al [21] and Van Leeuwen et al [37] models. In section 3 we demonstrate that there is excellent agreement between the continuum and discrete models, and that the crypt model permits homeostasis only for a limited range of values of a nondimensional parameter. Finally, in section 4 we conclude with a summary and discussion.

\section{Model development}

\subsection{Discrete model}

Most spatial models of the crypt are discrete in nature, with each cell treated as an individual (e.g. [21, 37]). Cell motion is determined by neglecting inertial effects and balancing the forces exerted upon a given cell by its neighbours with a drag force that is assumed to originate from both cell-matrix and internal cell friction; cell-cell friction is neglected. When cell division occurs, a new daughter cell is introduced adjacent to the mother cell. For example, Meineke et al [21] developed a crypt model in a cylindrical geometry in which the forces between cells were modelled via linear springs. Stem cells, positioned at the base of the crypt, were assumed to produce 
transit cells whose progeny further differentiate as they migrate up the crypt. The cell proliferation rates were estimated using data from murine intestinal crypts (e.g. stem cells divide at half the rate of transit cells).

Meineke et al [21] and Van Leeuwen et al [37] consider an off-lattice model in which the two basic properties of elastic repulsion and adhesive attraction between neighbouring cells are described by a linear force law. Cells are assumed to move on a fixed basal geometry; thus, the feedback between cell proliferation in the epithelial layer and deformation of the underlying mesenchyme is neglected. We note that these deformations were considered by Drasdo and Loeffler [4]. Applying Newton's second law in the over-damped limit, the equation of motion of the $n$th cell is given by

$$
\frac{\mathrm{d} \mathbf{r}_{n}}{\mathrm{~d} t}=\alpha \sum_{m}\left(\left|\mathbf{r}_{m}-\mathbf{r}_{n}\right|-a\right) \frac{\mathbf{r}_{m}-\mathbf{r}_{n}}{\left|\mathbf{r}_{m}-\mathbf{r}_{n}\right|},
$$

where $\mathbf{r}_{n}$ is the coordinate vector of the $n$th cell, the sum is taken over the $n$th cell's neighbours, $\alpha$ is the ratio of the spring constant to the cell viscosity and $a$ is the resting spring length.

In order to simulate cell motion in a crypt-like geometry, equation (1) is solved on the surface of a cylinder, which, for computational purposes, is unwrapped yielding a rectangular domain with periodic boundary conditions. It is assumed that cells can only leave the crypt via cell shedding or apoptosis at the top of the crypt; hence, the bottom of the crypt $(y=0)$ is modelled by imposing a 'hard wall' boundary condition while at the top of the crypt $(y=L)$, where cells are extracted into the intestinal lumen, it is assumed that cells experience zero stress. Nearest neighbours are defined using the Delaunay triangulation. The difference between the discrete model considered in this paper and those of Meineke et al [21] and Van Leeuwen et al [37] is that we assume a much simpler model of cell proliferation and differentiation: cell proliferation occurs in a spatially restricted region of the crypt $\left(y<y_{\text {Crit }}\right)$ with a mean cell cycle period, $T_{C}$, which can be interpreted as the average cell cycle period in the proliferative region (i.e. the average period of stem and transit cells). This simple spatial restriction of cell proliferation captures the observation that morphogen gradients, such as Wnt, restrict cell proliferation to the lower end of the crypt. Upon cell division, a daughter cell is placed in a random direction a short distance away from the mother cell. Simulations are performed using the Chaste computational infrastructure [29, 30].

\subsection{Continuum model}

Making the assumption that the crypt is symmetric about its longitudinal axis and hence that cell dynamics can be described using a 1D model, equation (1) can be coarse-grained in the continuum limit to the nonlinear diffusion equation

$$
\frac{\partial q}{\partial \tau}=\frac{\partial}{\partial y}\left(\frac{\alpha}{q^{2}} \frac{\partial q}{\partial y}\right)
$$

where $y$ is the spatial coordinate and $q(y, \tau)$ is the cell number density along the crypt axis [24]. A cell number density of $q=1 / a$ corresponds to the springs connecting neighbouring cells being at equilibrium while larger densities correspond to cell compression. We note that, rather than representing the active migration of cells, the nonlinear diffusive flux in the continuum model represents the passive effect of forces that neighbouring cells exert upon one another via their connecting springs (see appendix B).

Cell proliferation can be accounted for in this continuum description by the inclusion of a source term and the governing equation for cell number density is then given by

$$
\frac{\partial q}{\partial \tau}=\frac{\partial}{\partial y}\left(\frac{\alpha}{q^{2}} \frac{\partial q}{\partial y}\right)+H\left(y_{\text {Crit }}-y\right) \frac{\ln 2}{T_{C}} q,
$$

where $H(\cdot)$ denotes the Heaviside function. At the bottom of the crypt, the 'hard wall' boundary condition in the discrete simulations becomes

$$
\left.\frac{\partial q}{\partial y}\right|_{y=0}=0
$$

while at the top of the crypt $(y=L)$, the no-stress boundary condition becomes

$$
q(L, \tau)=\frac{1}{a},
$$

where $a$ is the natural spring length and $L$ is the crypt height, i.e. the cells being shed at the top of the crypt have equilibrium density. The initial conditions are taken to be

$$
q(y, 0)=q_{0}(y) .
$$

A schematic illustration of the model is presented in figure 1.

One of the benefits of transforming from a discrete to a continuum description of cell dynamics along the crypt axis is that analysis of the continuum model can help develop insight into governing mechanisms that determine coarsegrained features of the underlying simulations. For example, upon defining the cell flux along the crypt axis to be $J=v q$, where $v$ is the cell velocity field, we obtain, upon comparison with the flux term in equation (2), that

$$
v(y)=-\frac{\alpha}{q^{3}} \frac{\partial q}{\partial y} .
$$

Moreover, this expression can be used to calculate $T_{\text {Clear }}$, the time for a cell to reach the top of the crypt given an initial starting position, $y_{1}$, i.e.

$$
T_{\text {Clear }}=\int_{y_{1}}^{L} \frac{1}{v} \mathrm{~d} y .
$$

\subsection{Nondimensionalization}

We nondimensionalize equations (3)-(6) as follows:

$$
\hat{y}=\frac{y}{L}, \quad \hat{\tau}=\frac{\tau \ln 2}{T_{C}}, \quad \hat{q}=q a,
$$

where time has been nondimensionalized with the cell proliferation time-scale. Equations (3)-(6) are then given in the nondimensional form by

$$
\begin{gathered}
\beta \frac{\partial \hat{q}}{\partial \hat{\tau}}=\frac{\partial}{\partial \hat{y}}\left(\frac{1}{\hat{q}^{2}} \frac{\partial \hat{q}}{\partial \hat{y}}\right)+H\left(\hat{y}_{\text {Crit }}-\hat{y}\right) \beta \hat{q}, \\
\left.\frac{\partial \hat{q}}{\partial \hat{y}}\right|_{\hat{y}=0}=0,
\end{gathered}
$$




$$
\begin{gathered}
\hat{q}(1, \hat{\tau})=1, \\
\hat{q}(\hat{y}, 0)=\hat{q}_{0}(\hat{y}),
\end{gathered}
$$

where the nondimensional parameter $\beta=\ln 2 L^{2} / a^{2} T_{C} \alpha$ represents the ratio of the time-scale of cell movement along the length of the crypt axis to the cell division time-scale, and $\hat{y}_{\text {Crit }}=y_{\text {Crit }} / L$. For notational convenience we now drop the hatted notation.

The homeostatic behaviour of the crypt, which we are predominantly interested in from a biological perspective, is governed by the following equations:

$$
\begin{gathered}
\frac{\mathrm{d}}{\mathrm{d} y}\left(\frac{1}{q^{2}} \frac{\mathrm{d} q}{\mathrm{~d} y}\right)+H\left(y_{\text {Crit }}-y\right) \beta q=0, \\
\left.\frac{\mathrm{d} q}{\mathrm{~d} y}\right|_{y=0}=0, \\
q(1)=1,
\end{gathered}
$$

with the nondimensional crypt clearance time given by

$$
\hat{T}_{\text {Clear }}=-\int_{\hat{y}_{1}}^{1} \beta \hat{q}^{3}\left(\frac{\mathrm{d} \hat{q}}{\mathrm{~d} \hat{y}}\right)^{-1} \mathrm{~d} \hat{y} .
$$

Equations (14)-(16) were solved numerically using finite differences on a fixed equally spaced mesh containing $N_{\text {Nod }}$ grid points. Spatial derivatives were approximated using central differences. The resulting system of nonlinear difference equations was solved using the nonlinear leastsquares iterative solver 'fsolve' in Matlab. Solutions were checked for convergence and accuracy by considering finer spatial discretizations and reducing solver tolerances. We present the results below.

\section{Model analysis}

\subsection{Steady-state analysis}

In order to validate the use of the one-dimensional continuum model to describe cell behaviour in the two-dimensional discrete crypt, we have performed a range of simulations of the discrete model, as described in section 2.1, using different numerical values for the model parameters $\alpha, y_{\text {Crit }}$ and $T_{C}$. Simulations were run until dynamic equilibrium was attained ( $>200 \mathrm{~h}$ ) and cell velocities and densities were time averaged over 100 further hours of simulation time. For a given parameter set the numerical solution of equations (14)-(16) was calculated in order to determine the cell density profile along the crypt axis while the cell velocity field was calculated using equation (7). In figure 2 we present a representative sample of the results.

A notable feature of the discrete simulations is that the crypt does not always tend to a dynamic equilibrium. In figure 3 we present a plot of total cell number in the crypt against time for different values of the parameter $\alpha$. For small enough $\alpha$ the rate at which cells are produced at the bottom of the crypt is greater than the rate at which they can be removed via passive migration, the total cell number in the crypt grows in an unbounded manner and the cell density diverges leading to biologically unrealistic cell densities and inter-cell forces.

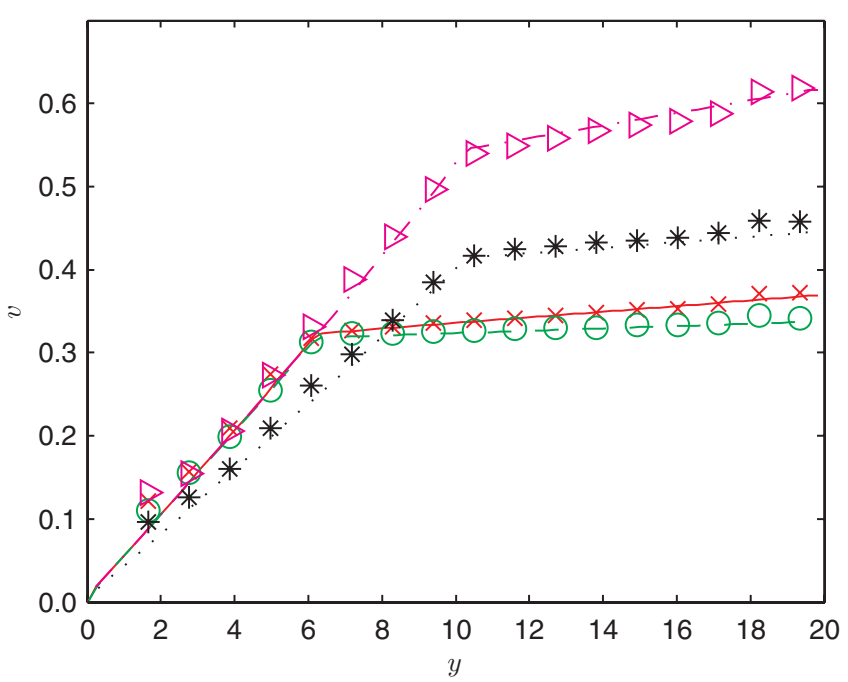

(a)

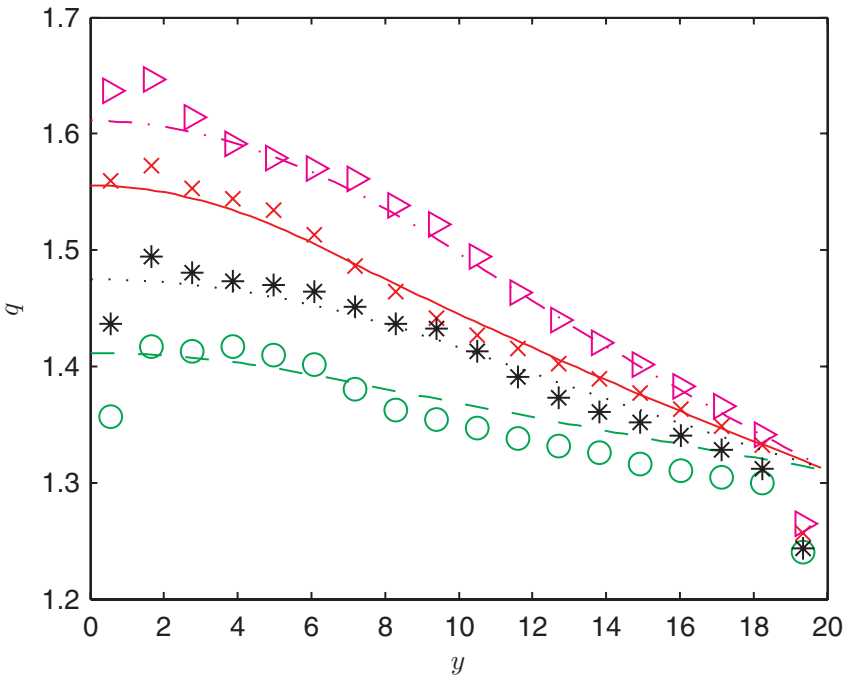

(b)

Figure 2. A comparison of discrete (markers) and continuum (lines) model solutions for a range of different parameter values. (a) Cell velocities, $v(y)$, plotted against crypt height, $y$. (b) Cell densities, $q(y)$, plotted against crypt height, $y$. Parameter values: $\left(\alpha, y_{\text {crit }}\right.$, $\left.T_{C}\right)=(40,6.3,14)$ (solid lines and crosses); $\left(\alpha, y_{\text {crit }}, T_{C}\right)=(80$, $6.3,14)$ (dashed lines and circles); $\left(\alpha, y_{\text {crit }}, T_{C}\right)=(50,10.3,14)$ (dot-dashed lines and triangles); $\left(\alpha, y_{\text {crit }}, T_{C}\right)=(50,10.3,18)$ (dotted lines and stars).

This dependence of dynamic equilibrium on the numerical value of the spring stiffness can be explained by analysis of the continuum model.

\subsection{Parameter space analysis}

In order to analyse steady-state behaviour in the continuum model we separate the crypt into proliferative (A) and nonproliferative (B) regions, where $y<y_{\text {Crit }}$ and $y>y_{\text {Crit }}$, respectively. In region $\mathrm{B}$, where there is no proliferation, the cell density profile is given by

$$
q_{B}(y)=\frac{1}{C_{1}(1-y)+1},
$$

where $C_{1}$ is an integration constant and the boundary condition (12) has been imposed. In region A we can find bounds for the 


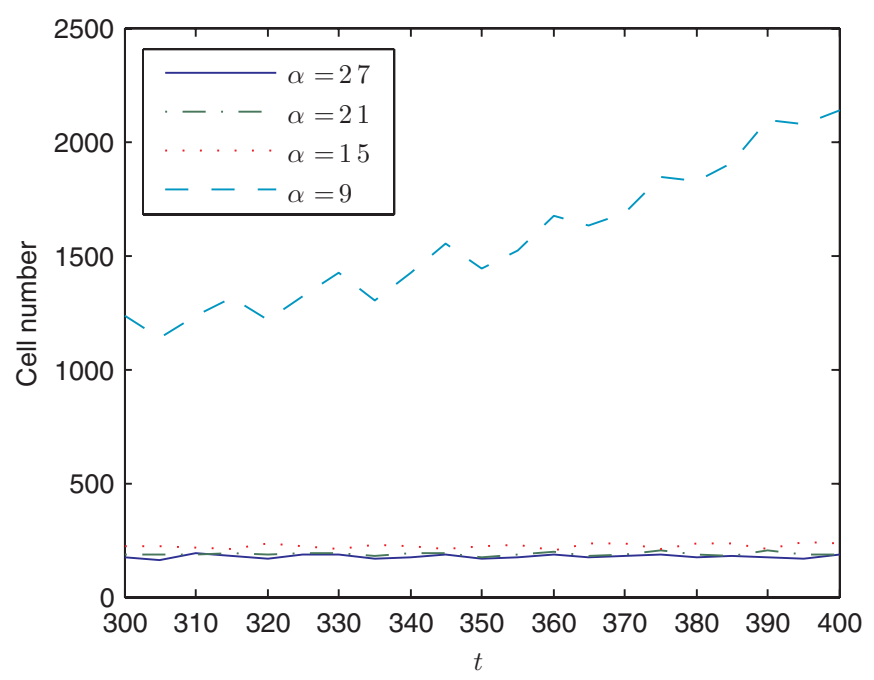

Figure 3. The total number of cells in the discrete simulation model is plotted against time when $\alpha=27,21,15,9$ (solid, dot-dashed, dotted and dashed lines, respectively). In these simulations $T_{C}=14$ and $y_{\text {Crit }}=\frac{1}{3}$. Simulations were initialized $(t=0)$ from an equilibrium configuration (data not shown).

derivative $\frac{\mathrm{d} q}{\mathrm{~d} y}$ (see appendix A) and hence obtain that there is no homeostatic solution to the crypt model when the inequality

$$
\beta>\frac{1}{y_{\text {Crit }}\left(2-y_{\text {Crit }}\right)}
$$

holds.

Considering numerical solutions of equations (14)-(16) we demonstrate that there is a limited region of $\left(\beta, y_{\text {Crit }}\right)$ parameter space that yields homeostatic solutions (see figure $4(a)$ ). When $\beta$ is large enough, the cell proliferation rate is much larger than the rate at which cells can move up the crypt and a steady-state solution is not attainable (the number of cells tends to infinity). The region of parameter space in which homeostatic solutions are possible is bounded by inequality (19). In figure $4(b)$ corresponding solutions of the discrete model show good qualitative agreement with both numerical solutions of the continuum models and inequality (19).
In dimensional units a necessary condition for homeostasis is therefore

$$
\alpha>\frac{\log 2 L^{4}}{a^{2} T_{C} y_{\text {Crit }}\left(2-y_{\text {Crit }}\right)} .
$$

Thus, assuming fixed values for the other parameters, there is a critical value of $\alpha$ below which the homeostatic solution is lost. This is precisely the behaviour presented in figure 3. A similar argument can be applied to other model parameters. For example, in longer crypts, as the cells have a further distance to travel to the top of the crypt, the cell movement timescale increases relative to the proliferation time-scale and a threshold exists beyond which the homeostatic solution is lost. The inequality (20) can therefore be used to guide parameter choice in the simulation of longer crypts (e.g. in other regions of the intestine).

The breakdown in crypt homeostasis is dependent on the interplay between the processes of movement and proliferation in the crypt. Outside the homeostatic range of parameter space, high cell densities at the bottom of the crypt yield a small diffusion coefficient, cell movement decreases and cells cannot escape from the proliferative zone. As cell proliferation depends linearly on cell density, there is positive feedback in the system, the cell density increases further and so on. Hence the loss of the homeostatic solution is dependent on the forms of the nonlinear diffusion coefficient and the cell proliferation rate. Both of these assumptions would have to be validated in a given experimental context before one could speculate on the physiological relevance of the breakdown of crypt homeostasis in the model.

\subsection{Dimensional analysis}

In this section we demonstrate using data from murine small intestinal crypts how results from the continuum model can be used to efficiently determine biologically relevant regions of parameter space. The key experimental data, taken from [31] and presented in table 1, are compared with the following model quantities: (a) the number of cells in the proliferative region of the crypt $\left(\int_{0}^{y_{\text {Crit }}} q(y) \mathrm{d} y\right)$; (b) the time for crypt renewal (equation (8)); (c) the total cell number in the crypt

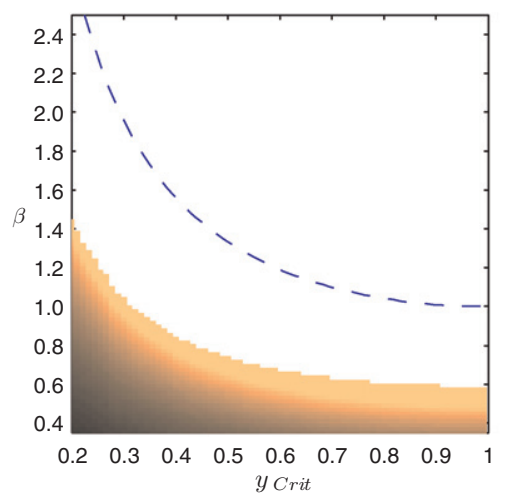

(a)
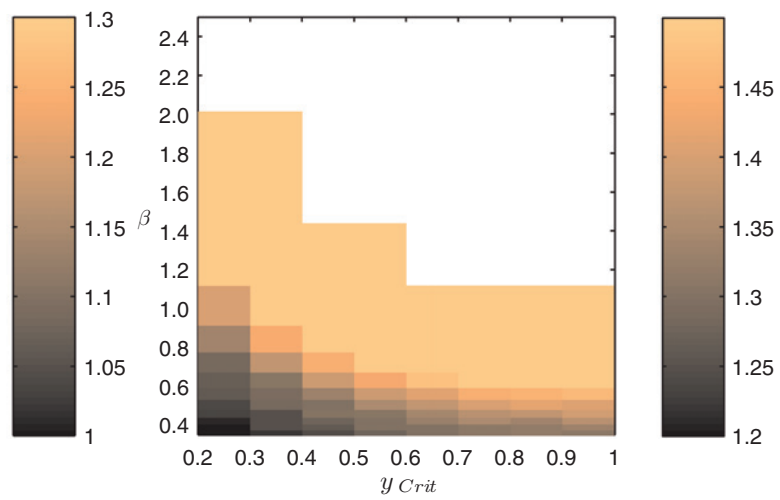

$(b)$

Figure 4. Average densities in the continuum (a) and discrete $(b)$ models are plotted against the nondimensional parameters $\beta$ and $y_{\text {Crit }}$. The dashed line denotes the upper bound presented in inequality (19). 


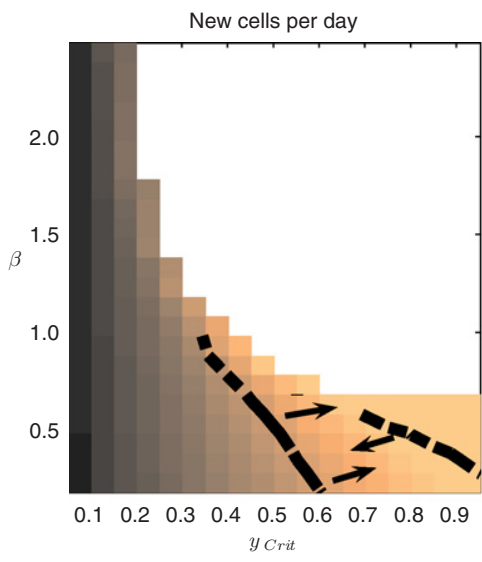

(a)

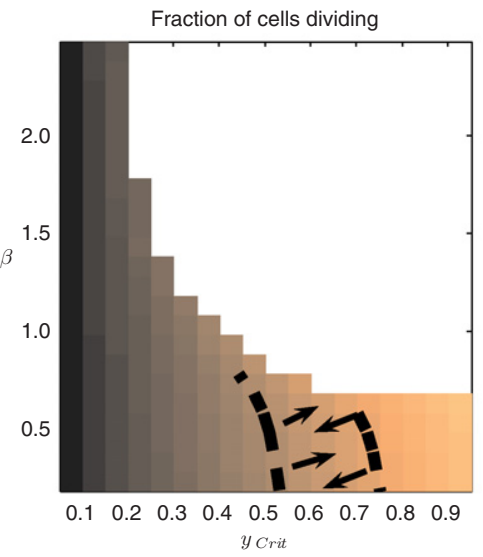

(c)
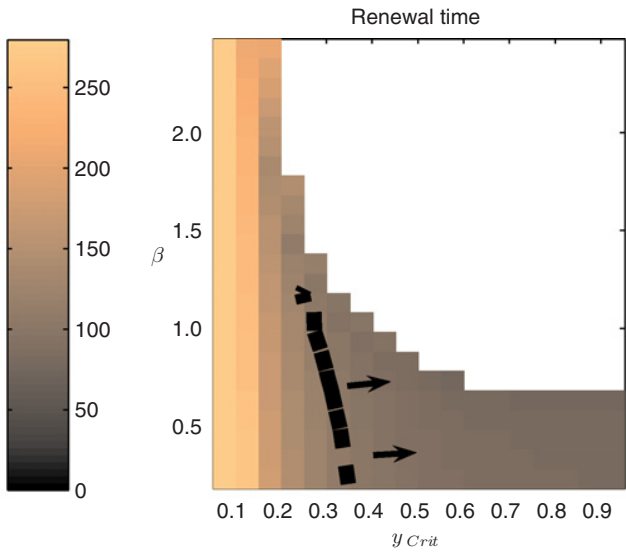

(b)
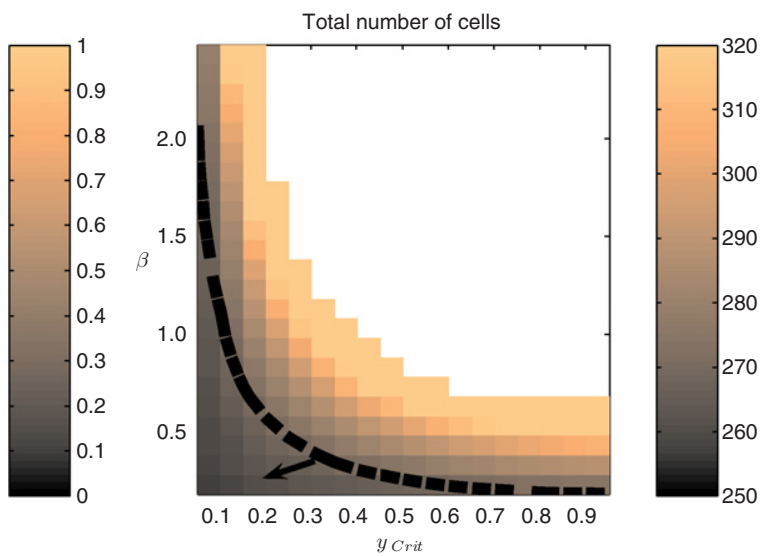

$(d)$

Figure 5. Determining what values of the model parameters $\beta$ and $y_{\text {Crit }}$ yield biologically reasonable crypt behaviours. (a) New cells per day, $(b)$ crypt renewal time, $(c)$ fraction of cells dividing in the crypt and (d) total cell number are plotted for different values of the two model parameters $\beta$ and $y_{\text {Crit }}$. The shaded bars denote numerical values of the respective variables. The contours (dashed lines) enclose that region of parameter space that lies within $20 \%$ of the experimentally quoted values presented in table 1 .

Table 1. Experimentally known quantities in the murine small intestinal crypt taken from [31] with the exception of the crypt renewal time which is taken from [28]. Note that crypt labelling experiments typically show $\sim 25$ labelled cells along a section but that Potten and Loeffler [31] have measured the crypt to have a height of 16 cell diameters. The extra nine cells are an experimental artefact resulting from the section width and packing arrangements [31].

\begin{tabular}{ll}
\hline Quantity & Description \\
\hline Crypt length $(L)$ & 16 cell diameters \\
Crypt circumference & 16 cell diameters \\
Number of cells in the crypt & 250 \\
Fraction of proliferative cells & $\frac{2}{3}$ \\
Cells produced per day & 300 \\
Cell velocity at the top $(v(L))$ & 0.75 cell positions per day \\
Number of stem cells $\left(N_{S}\right)$ & $1-16$ \\
Number of transit cells $\left(N_{T}\right)$ & 160 \\
Number of Paneth cells $\left(N_{P}\right)$ & 30 \\
Stem cell cycle period $\left(T_{S T}\right)$ & $24 \mathrm{~h}$ \\
Transit cell cycle period $\left(T_{\mathrm{Tr}}\right)$ & $12 \mathrm{~h}$ \\
Crypt renewal time $\left(T_{\mathrm{Clear}}\right)$ & $2-3$ days \\
\hline
\end{tabular}

$\left(\int_{0}^{L} q(y) \mathrm{d} y\right)$ and (d) the fraction of proliferating cells (the ratio of the number of cells in the proliferative region to the total number of cells).
In order to estimate the parameter $T_{C}$, which represents the average cell cycle period in the proliferative region of a homeostatic crypt, we assume that the proliferative region is occupied at some time $t_{0}$ by $N_{P}$ non-proliferative, $N_{S}$ stem and $N_{T}$ transit cells. Neglecting cell motion, we estimate that at $t_{0}+T_{C}$ there are

$$
N_{P}+N_{S} \mathrm{e}^{\frac{\ln 2}{T_{\mathrm{St}}} T_{C}}+N_{T} \mathrm{e}^{\frac{\ln 2}{T_{\mathrm{Tr}}} T_{C}}
$$

mother and daughter cells, where $T_{\mathrm{St}}$ and $T_{\mathrm{Tr}}$, the stem and transit cell cycle periods, respectively, are 24 and $12 \mathrm{~h}$ (see table 1). Setting

$$
2\left(N_{P}+N_{S}+N_{T}\right)=N_{P}+N_{S} \mathrm{e}^{\frac{\ln 2}{T_{\mathrm{St}}} T_{C}}+N_{T} \mathrm{e}^{\frac{\ln 2}{T_{\mathrm{Tr}}} T_{C}},
$$

we can solve for $T_{C}$ (assuming $T_{\mathrm{Tr}}=\frac{1}{2} T_{\mathrm{St}}$ ) to obtain that the average doubling time in the proliferative region of the crypt is

$T_{C}=\frac{T_{\mathrm{St}}}{\ln 2} \ln \left(\frac{-N_{S}+\sqrt{N_{S}^{2}+4\left(N_{P}+2 N_{S}+2 N_{T}\right) N_{T}}}{2 N_{T}}\right)$,

which upon substitution for the experimentally estimated values yields (see table 1)

$$
T_{C} \sim 13.8 \mathrm{~h} .
$$




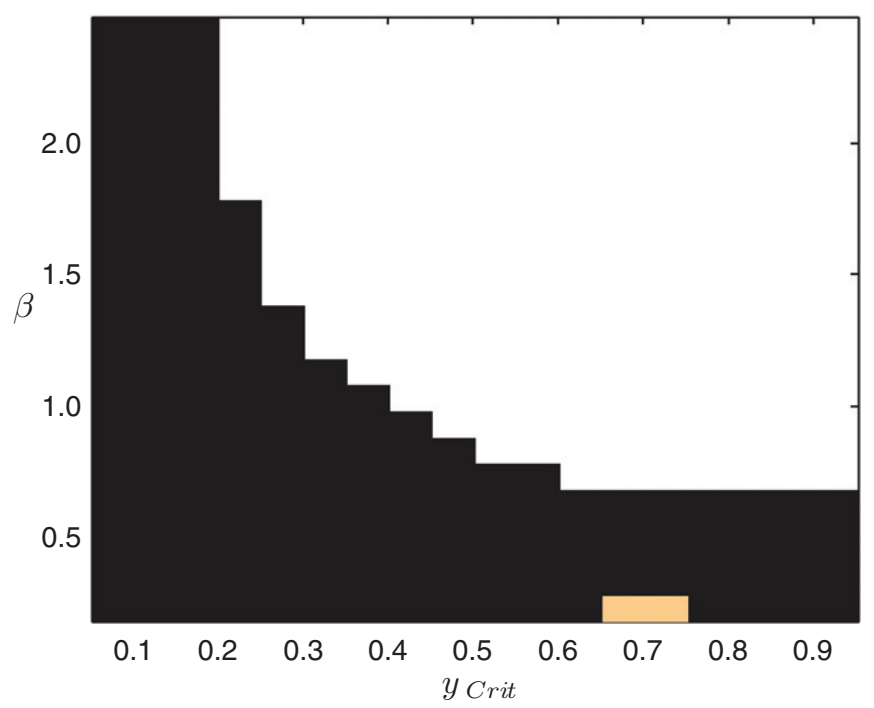

Figure 6. The narrow region of parameter space (light region) that is consistent with the data presented in table 1 is determined by combining results presented in figure 5, i.e. $y_{\text {Crit }} \sim 0.7, \beta<0.3$.

In figure 5 we compare the experimentally known data listed in table 1 with results from the continuum model. As error estimates are not provided for most of the given data, approximate regions of parameter space in which model output is within $20 \%$ of the estimated experimental value are highlighted (solid lines and arrows). In figures 5(a), (c) and $(d)$, the number of cells produced in the crypt, the fraction of cells that are dividing and the total number of cells in the crypt, respectively, increase with the size of the proliferative region or the cell division rate, as expected. In figure $5(b)$ the crypt renewal time (i.e. time taken by a cell near the bottom of the crypt to reach the top) decreases with $y_{\text {Crit }}$ and increases with $\beta$.

In figure 6 we combine the results presented in figure 5 in order to define a region of parameter space that produces results that are in agreement with the experimental data presented in table 1. These results suggest that a reasonable choice for the model parameters is $y_{\text {Crit }} \sim \frac{2}{3}$ and $\beta<0.3$ for a murine small intestinal crypt. Recalling that

$$
\beta=\frac{\ln 2 L^{2}}{a^{2} \alpha T_{C}},
$$

and substituting for $L=16$ and $T_{C}=13.8$, we obtain

$$
\alpha>\frac{\ln 2 L^{2}}{a^{2} \beta T_{C}}=18 .
$$

In previous models, Meineke et al [21] and Van Leeuwen et al [37] fitted $\alpha=30$ based upon the resulting simulation output.

\section{Conclusions}

In this study we have developed a one-dimensional continuum model of the crypt based upon the coarse-graining of previous discrete models. By comparing cell velocity and density fields along the crypt axis, we have demonstrated that the continuum model is in good agreement with corresponding discrete model simulations. The major benefit of this approach is that the continuum model can be analysed quickly and easily and a broad parameter space can be explored, yielding insights regarding the underlying discrete simulations. We envisage that results from the continuum model can be used as a guide in the design and interpretation of future discrete simulations of intestinal crypts.

Following the work of Meineke et al [21] and Van Leeuwen et al [37], we have assumed that (a) cells interact via a linear force law and (b) cells move on a fixed cylindrical geometry. The linear force law assumption represents the simplest description in an off-lattice framework of the elastic repulsion and adhesive attraction between neighbouring cells. The advantage of using nonlinear force laws, such as the Hertz or Jones-Kenall-Roberts (JKR) [15] models (see e.g. $[3,5,8])$, is that in these models the parameters are, in principle, experimentally accessible. However, we note that it is thought that once characteristic biological features and mechanisms, such as cell adhesion and repulsion, are satisfactorily represented in a discrete simulation framework, qualitative and many quantitative features of simulation results appear to be independent of the precise model details [8, 9]. With regard to the assumption of a fixed geometry, we note that healthy crypts can undergo morphological changes, such as crypt fission, over time-scales of the order of tens of years [12]. The frequency of fission events can increase dramatically in cancerous tissue and fission is thought to be a key mechanism by which mutations can spread throughout the gut from a single crypt. Clearly, the assumption of a fixed geometry needs to be relaxed if one wishes to couple proliferation in the crypt to morphological changes in crypt structure $[4,6]$. This more complicated problem is a current topic of research in our group.

We have assumed that the net effect of molecular regulation of cell proliferation is to define a threshold proliferation height, $y_{\text {Crit }}$, above which proliferation does not occur. This approach allows us to classify model behaviour in terms of the two nondimensional parameters $\beta$ and $y_{\text {Crit }}$ : when the cell division time-scale is sufficiently decreased relative to that of cell movement along the crypt axis, the homeostatic solution is lost. This result was verified by considering simulations of the discrete model in different regions of parameter space. We note that, in reality, spatial regulation of cell proliferation along the crypt axis depends on a range of factors, such as extracellular signalling and refer the interested reader to [25] where we demonstrate how the parameter $y_{\text {Crit }}$ can be related to Wnt pathway regulation.

Whether or not the blow-up uncovered by the continuum model analysis is biologically relevant is dependent on the accuracy of the underlying assumptions in the discrete simulations: cells interact via a linear force law and divide at an exponential rate in the proliferative region of the crypt. We note that one way to remove the blow-up in our model framework is to introduce feedbacks that modify cell proliferation and/or movement. For example, by considering a hard-wall-like potential (e.g. [19]), cell densities can never exceed some pre-specified threshold density. Alternatively, Murray et al [25] assume that cell proliferation is regulated by 
contact inhibition and the inclusion of a logistic growth term ensures that cell densities cannot reach the threshold required for the blow-up. We note that the assumption that the feedback between cell proliferation and geometry is negligible would become invalid in the limit of excessive densities in the crypt.

We have identified a small region of non-dimensional parameter space which yields numerical results consistent with experimental data from murine intestinal crypts. The advantage of the continuum model analysis is that we can derive expressions for experimentally measurable quantities, such as the crypt renewal time; hence, parameter sweeps can be performed much more efficiently than by using the corresponding discrete model. We note that a similar approach could be applied to data from other species or regions of the intestines.

Finally, we highlight the need to develop both discrete and continuum models for multiscale systems. As exemplified in this study, the continuum approach allowed us to generalize the description of the intestinal crypt model and to classify model behaviour. However, when, for example, an understanding of the behaviour of small subpopulations of cells is required, such as in the study of cell lineages, simulation of the discrete model is essential as the continuum model becomes invalid. In summary, while the discrete simulation approach can allow us to answer specific questions, the continuum model can be used to develop insight into the coarse-grained behaviour of the underlying simulations.

\section{Acknowledgments}

PJM, AW, AGF and CME acknowledge the support of the Engineering and Physical Sciences Research Council through the Integrative Biology Project (GR/S72023/01). PJM, AW and AGF also acknowledge training provided by the Oxford University Life Sciences Interface Doctoral Training Centre (LSI DTC). AGF is funded through the OCISB project (BB/D020190/1). Part of this work (PJM and PKM) was supported by NIH grant U56CA113004 from the National Cancer Institute, and the British Council through PMI2. PKM was partially supported by a Royal Society-Wolfson Research Merit Award. PJM thanks Mark Chaplain and David Gavaghan for helpful advice.

\section{Appendix A. Deriving the crypt inequality}

Making the change of variable $q=1 / u$ the system (14)-(16) becomes

$$
\begin{gathered}
\frac{\mathrm{d}^{2} u}{\mathrm{~d} y^{2}}=\beta H\left(y_{\text {Crit }}-y\right) u^{-1}, \quad 0 \leqslant y \leqslant 1, \\
\frac{\mathrm{d} u}{\mathrm{~d} y}(0)=0, \\
u(1)=1 .
\end{gathered}
$$

The region $y_{\text {Crit }} \leqslant y \leqslant 1$

In the region $y_{\text {Crit }} \leqslant y \leqslant 1$, equation (A.1) reduces to

$$
\frac{\mathrm{d}^{2} u}{\mathrm{~d} y^{2}}=0 \text {. }
$$

The solution to this equation that satisfies boundary condition (A.3) is given by

$$
u(y)=1-A(1-y), \quad y_{\text {Crit }} \leqslant y \leqslant 1,
$$

for some constant $A$. Note that since we are interested only in solutions $u \geqslant 0$, the right-hand side of equation (A.1) is nonnegative. This, together with boundary conditions (A.2) and (A.3), means that the solution $u$ must increase monotonically with $y$, attaining its maximum value at $y=1$. In particular, we have that $0 \leqslant u\left(y_{\text {Crit }}\right) \leqslant 1$. Using the above expression for $u$ and rearranging this inequality, we find that the unknown constant $A$ must satisfy

$$
0 \leqslant A \leqslant \frac{1}{1-y_{\text {Crit }}} .
$$

The region $0 \leqslant y<y_{\text {Crit }}$

In the region $0 \leqslant y<y_{\text {Crit }}$, equation (A.1) reduces to

$$
u \frac{\mathrm{d}^{2} u}{\mathrm{~d} y^{2}}=\beta
$$

This equation is nonlinear and cannot be solved analytically; however, we can make further progress as follows. We rewrite equation (A.7) as

$$
\frac{\mathrm{d}}{\mathrm{d} y}\left(u \frac{\mathrm{d} u}{\mathrm{~d} y}\right)-\left(\frac{\mathrm{d} u}{\mathrm{~d} y}\right)^{2}=\beta,
$$

and integrate with respect to $y$, imposing boundary condition (A.2). We obtain the integro-differential equation

$$
u \frac{\mathrm{d} u}{\mathrm{~d} y}-\int_{0}^{y}\left(\frac{\mathrm{d} u}{\mathrm{~d} x}\right)^{2} \mathrm{~d} x=\beta y, \quad 0 \leqslant y \leqslant y_{\text {Crit }} .
$$

This may be rewritten as

$$
\frac{\mathrm{d}}{\mathrm{d} y}\left(\frac{1}{2} u^{2}\right)-\int_{0}^{y}\left(\frac{\mathrm{d} u}{\mathrm{~d} x}\right)^{2} \mathrm{~d} x=\beta y
$$

and integrated with respect to $y$, once more imposing boundary condition (A.2), to give

$$
\begin{aligned}
\frac{1}{2}\left(u(y)^{2}-\right. & \left.u(0)^{2}\right)-\int_{0}^{y} \int_{0}^{\xi} \\
& \times\left(\frac{\mathrm{d} u}{\mathrm{~d} \zeta}\right)^{2} \mathrm{~d} \zeta \mathrm{d} \xi=\frac{1}{2} \beta y^{2}, \quad 0 \leqslant y \leqslant y_{\text {Crit }} .
\end{aligned}
$$

Evaluating this last equation at $y=y_{\text {Crit }}$ and imposing continuity of $u$ there (so that $u\left(y_{\text {Crit }}\right)=1-A\left(1-y_{\text {Crit }}\right)$ ), we obtain

$$
\begin{gathered}
\frac{1}{2}\left(\left(1-A\left(1-y_{\text {Crit }}\right)\right)^{2}-u(0)^{2}\right)-\int_{0}^{y_{\text {Crit }}} \int_{0}^{\xi} \\
\times\left(\frac{\mathrm{d} u}{\mathrm{~d} \zeta}\right)^{2} \mathrm{~d} \zeta \mathrm{d} \xi=\frac{1}{2} \beta y_{\text {Crit }}^{2} .
\end{gathered}
$$


As previously identified, $u$ must increase monotonically from $y=0$ to $y=y_{\text {Crit }}$. Furthermore, if we impose boundary condition (A.2) and the matching condition $\mathrm{d} u / \mathrm{d} y\left(y_{\text {Crit }}\right)=A$, we obtain the inequality

$$
0 \leqslant \frac{\mathrm{d} u}{\mathrm{~d} y} \leqslant A \text { for } 0 \leqslant y \leqslant y_{\text {Crit }} ;
$$

hence,

$$
0 \leqslant \int_{0}^{y_{\text {Crit }}} \int_{0}^{\xi}\left(\frac{\mathrm{d} u}{\mathrm{~d} \zeta}\right)^{2} \mathrm{~d} \zeta \mathrm{d} \xi \leqslant \frac{1}{2} A^{2} y_{\text {Crit }}^{2}
$$

and, using (A.12), we obtain

$$
\begin{aligned}
& \frac{1}{2}\left(\left(1-A\left(1-y_{\text {Crit }}\right)\right)^{2}-u(0)^{2}\right)-\frac{1}{2} A^{2} y_{\text {Crit }}^{2} \\
& \leqslant \frac{1}{2} \beta y_{\text {Crit }}^{2} \leqslant \frac{1}{2}\left(\left(1-A\left(1-y_{\text {Crit }}\right)\right)^{2}-u(0)^{2}\right) .
\end{aligned}
$$

Next, note that in the region $0 \leqslant y \leqslant y_{\text {Crit }}$, the solution $u$ must lie above $1-A(1-y)$; this results from the fact that in this region, the right-hand side of equation (A.1) is strictly positive. This means that we must have

$$
1-A \leqslant u(0) \leqslant 1-A\left(1-y_{\text {Crit }}\right),
$$

which we can then use to eliminate $u(0)$ from inequality (A.15). We obtain

$0 \leqslant \frac{1}{2} \beta y_{\text {Crit }}^{2} \leqslant \frac{1}{2}\left(\left(1-A\left(1-y_{\text {Crit }}\right)\right)^{2}-(1-A)^{2}\right)$,

and hence

$$
0 \leqslant \beta \leqslant \frac{A}{y_{\text {Crit }}}\left(2-A\left(2-y_{\text {Crit }}\right)\right) .
$$

The last step is to note that the function $f(A)=\frac{A}{y_{\text {Crit }}}(2-$ $\left.A\left(2-y_{\text {Crit }}\right)\right)$ attains its maximum value of $1 / y_{\text {Crit }}\left(2-y_{\text {Crit }}\right)$ at the point $A=1 /\left(2-y_{\text {Crit }}\right)$, which lies within the interval (A.6). Hence, we obtain

$$
\beta \leqslant \frac{1}{y_{\text {Crit }}\left(2-y_{\text {Crit }}\right)}
$$

\section{Appendix B. Coarse-graining a two-dimensional spring-based model}

\section{B.1. The one-dimensional case}

Murray et al [24] consider a one-dimensional chain of cells that interact via a linear force law. Applying Newton's second law in the overdamped limit, the equation of motion for the $i$ th cell is given by

$$
\frac{\mathrm{d} r_{i}(t)}{\mathrm{d} t}=\alpha\left(r_{i-1}(t)-2 r_{i}(t)+r_{i+1}(t)\right), \quad i=2, \ldots, N-1,
$$

where the parameter $\alpha$ is the ratio of the spring constant, $k$, to the cell viscosity, $\eta$, i.e. $\alpha=\frac{k}{\eta}$. In the continuum limit

$$
\frac{\partial r}{\partial t}=\alpha \frac{\partial^{2} r}{\partial i^{2}}
$$

where $r$ is treated as a continuous function of $i$ such that

$$
r(i, t)=r_{i}(t) \text {. }
$$

Equation (B.2) can be reformulated such that cell number density is the dependent variable by making a coordinate transformation from the old independent variables $i$ and $t$ to the new independent variables $r$ (the dependent variable in the old coordinate system) and $\tau$ (time). Subsequently differentiating with respect to $r$ we obtain the partial differentiation equation

$$
\frac{\partial q}{\partial \tau}=\frac{\partial}{\partial r}\left(\frac{\alpha}{q^{2}} \frac{\partial q}{\partial r}\right)
$$

where

$$
q(r, \tau)=\frac{\partial,(r, \tau)}{\partial r}
$$

The no-stress boundary condition can be reformulated by introducing an image cell at the boundary such that $r_{N+1}=$ $r_{N}+a$, which transforms in the continuum limit to $q(L)=1 / a$. For further information we refer the interested reader to [24].

\section{B.2. The two-dimensional case}

Using mitochondrial DNA staining experiments, Taylor et al [35] and McDonald et al [20] have observed that cell motion in a healthy crypt is directed predominantly along the crypt axis. Whilst this observation provides justification for the assumption of one spatial dimension in equation (2), below we describe how the one-dimensional continuum model can be derived from a discrete model of cell dynamics in two spatial dimensions.

In two spatial dimensions the equation of motion of the $n$th cell, positioned at $\mathbf{r}_{\mathbf{n}}$, is

$$
\frac{\mathrm{d} \mathbf{r}_{n}}{\mathrm{~d} t}=\alpha \sum_{m}\left(\left|\mathbf{r}_{m}-\mathbf{r}_{n}\right|-a\right) \frac{\mathbf{r}_{m}-\mathbf{r}_{n}}{\left|\mathbf{r}_{m}-\mathbf{r}_{n}\right|},
$$

where the sum is taken over a cell's neighbours and $\alpha$ is again the ratio of spring constant to cell viscosity. Defining $\mathbf{r}_{n}$ in Euclidean coordinates to be

$$
\mathbf{r}_{n}=x_{n} \mathbf{i}+y_{n} \mathbf{j},
$$

the equations of motion can be resolved along the $x$ and $y$ axes yielding

$$
\begin{aligned}
\frac{\mathrm{d} x_{n}}{\mathrm{~d} t} & =\alpha \sum_{m}\left(x_{m}-x_{n}\right)-\alpha a \sum_{m} \cos \theta_{m}, \\
\frac{\mathrm{d} y_{n}}{\mathrm{~d} t} & =\alpha \sum_{m}\left(y_{m}-y_{n}\right)-\alpha a \sum_{m} \sin \theta_{m},
\end{aligned}
$$

where $\theta_{m}$ is defined to be the angle that the centre of the $m$ th cell makes with respect to the centre of the $n$th and the $x$ axis in a local coordinate system where the $n$th cell is taken to be the origin. The first terms in equations (B.8) and (B.9) take a similar form to the equation of motion of a cell in the $1 \mathrm{D}$ case (see equation (B.1)). The second terms are nonlinear and explicitly couple the dynamics of the cells in the $x$ direction with those in the $y$ direction.

In simulations of the two-dimensional crypt model, a cell's neighbours are regularly distributed around it. Making this 
assumption in equations (B.8) and (B.9), the second terms on the right-hand sides are zero, hence we obtain that

$$
\frac{\mathrm{d} x_{n}}{\mathrm{~d} t}=\alpha \sum_{m}\left(x_{m}-x_{n}\right)
$$

and

$$
\frac{\mathrm{d} y_{n}}{\mathrm{~d} t}=\alpha \sum_{m}\left(y_{m}-y_{n}\right)
$$

In a similar manner to the one-dimensional case, we assume that the right-hand sides of equations (B.10) and (B.11) can be written as Laplacians in which the independent variables are $i$ and $j$. Again, analogously with the one-dimensional case, we make a coordinate transformation in independent variables from $i j$ to $x y$ with the cell number density given by the Jacobian determinant of the coordinate transformation, i.e.

$$
q_{n}(x, y, \tau)={\frac{\partial i_{n}}{\partial x}}_{\mid y \tau}{\frac{\partial j_{n}}{\partial y}}_{\mid x \tau}-{\frac{\partial i_{n}}{\partial y}{ }_{\mid x \tau}}_{\frac{\partial j_{n}}{\partial x}{ }_{\mid y \tau}} .
$$

We then define the symmetric connectivity tensor, $A_{n}$, to be

$$
A_{n}=\left(\begin{array}{cc}
\left(\frac{\partial j_{n}}{\partial y}\right)^{2}+\left(\frac{\partial i_{n}}{\partial y}\right)^{2} & -\frac{\partial j_{n}}{\partial x} \frac{\partial j_{n}}{\partial y}-\frac{\partial i_{n}}{\partial x} \frac{\partial i_{n}}{\partial y} \\
-\frac{\partial j_{n}}{\partial x} \frac{\partial j_{n}}{\partial y}-\frac{\partial i_{n}}{\partial x} \frac{\partial i_{n}}{\partial y} & \left(\frac{\partial j_{n}}{\partial x}\right)^{2}+\left(\frac{\partial i_{n}}{\partial x}\right)^{2}
\end{array}\right),
$$

and find that the governing equation for cell number density is of the form

$$
-\frac{1}{\alpha} \frac{\partial q_{n}}{\partial \tau}=\nabla \cdot\left(\nabla \frac{A_{n}}{q_{n}}\right)
$$

In order to derive a closed-form model in which $q_{n}$ is the only dependent variable a further assumption is required. Given the observation of axial symmetry in normal crypts $[20,35]$, we consider a particular case of equation (B.14) in which

$$
\frac{\partial i_{n}}{\partial y}=\frac{\partial j_{n}}{\partial x}=0
$$

such that the cell number density reduces to the separable form

$$
q_{n}(x, y, \tau)=\frac{\partial i_{n}(x, \tau)}{\partial x} \frac{\partial j_{n}(y, \tau)}{\partial y},
$$

and equation (B.13) simplifies to

$$
A_{n}=\left(\begin{array}{cc}
\left(\frac{\partial j_{n}}{\partial y}\right)^{2} & 0 \\
0 & \left(\frac{\partial i_{n}}{\partial x}\right)^{2}
\end{array}\right)
$$

Upon substitution of equation (B.17), equation (B.14) can be written as

$$
\frac{\partial q_{n}}{\partial \tau}=\alpha\left(\frac{\partial j_{n}}{\partial y}\right)^{2} \frac{\partial}{\partial x}\left(\frac{1}{q_{n}^{2}} \frac{\partial q_{n}}{\partial x}\right)+\alpha\left(\frac{\partial i_{n}}{\partial x}\right)^{2} \frac{\partial}{\partial y}\left(\frac{1}{q_{n}^{2}} \frac{\partial q_{n}}{\partial y}\right) .
$$

For notational convenience we define

$$
\begin{aligned}
& q_{n 1}(x, \tau)=\frac{\partial i_{n}}{\partial x}, \\
& q_{n 2}(y, \tau)=\frac{\partial j_{n}}{\partial y},
\end{aligned}
$$

which are the numbers of cells per unit length along the $x$ and $y$ axes, respectively, and substitution in equation (B.18) yields $\frac{\partial q_{n}}{\partial \tau}=\alpha q_{n 2}^{2} \frac{\partial}{\partial x}\left(\frac{1}{q_{n}^{2}} \frac{\partial q_{n}}{\partial x}\right)+\alpha q_{n 1}^{2} \frac{\partial}{\partial y}\left(\frac{1}{q_{n}^{2}} \frac{\partial q_{n}}{\partial y}\right)$.

Finally, assuming axial symmetry, $q_{n}$ is constant with respect to $x$, and equation (B.20) reduces to the $1 \mathrm{D}$ form

$$
\frac{\partial q_{n}}{\partial \tau}=\frac{\partial}{\partial y}\left(\frac{\alpha}{q_{n}^{2}} \frac{\partial q_{n}}{\partial y}\right) .
$$

We refer the interested reader to [23] for further details.

\section{References}

[1] Bienz M and Clevers H 2000 Linking colorectal cancer to Wnt signaling Cell 103 311-20

[2] Boman B M, Walters R, Fields J Z, Kovatich A J, Zhang T, Isenberg G A, Goldstein S D and Palazzo J P 2004 Colonic crypt changes during adenoma development in familial adenomatous polyposis: immunohistochemical evidence for expansion of the crypt base cell population Am. J. Pathol. 165 1489-98

[3] Byrne H M and Drasdo D 2009 Individual-based and continuum models of growing cell populations: a comparison J. Math. Biol. 58 657-87

[4] Drasdo D and Loeffler M 2001 Individual-based models to growth and folding in one-layered tissues: intestinal crypts and early development Nonlinear Anal. Theory Methods Appl. 47 245-56

[5] Drasdo D, Hoehme S and Block M 2007 On the role of physics in the growth and pattern formation of multi-cellular systems: what can we learn from individual-cell based models? J. Stat. Phys. 128 287-345

[6] Edwards C M and Chapman S J 2007 Biomechanical modelling of colorectal crypt budding and fission Bull. Math. Biol. 69 1927-42

[7] Fozard J A, Byrne H M, Jensen O E and King J R 2010 Continuum approximations of individual-based models for epithelial monolayers Math. Med. Biol. 27 39-74

[8] Galle J and Preziosi L 2009 Multiphase and individual cell-based models of tumour growth Mathematics, Developmental Biology and Tumour Growth: UIMP-RSME Lluis A. Santaló Summer School (Universidad Internacional Menéndez Pelayo, Santander, Spain, 11-15 September 2006 vol 492) p 103

[9] Galle J, Aust G, Schaller G, Beyer T and Drasdo D 2006 Individual cell-based models of the spatial-temporal organization of multicellular systems-achievements and limitations Cytometry A 69 704-10

[10] Gaspar C and Fodde R 2004 APC dosage effects in tumorigenesis and stem cell differentiation Int. J. Dev. Biol. 48 377-86

[11] Giles R H, van Es J H and Clevers H 2003 Caught up in a Wnt storm: Wnt signaling in cancer BBA-Rev. Cancer 1653 1-24

[12] Greaves L C et al 2006 Mitochondrial DNA mutations are established in human colonic stem cells, and mutated clones expand by crypt fission Proc. Natl Acad. Sci. 103714 
[13] Gregorieff A, Pinto D, Begthel H, Destrée O, Kielman M and Clevers H 2005 Expression pattern of Wnt signaling components in the adult intestine Gastroenterology $129626-38$

[14] Hanahan D and Weinberg R A 2000 The hallmarks of cancer Cell 100 57-70

[15] Johnson K L, Kendall K and Roberts A D 1971 Surface energy and the contact of elastic solids Proc. R. Soc. A 324 301-13

[16] Johnston M D, Edwards C M, Bodmer W F, Maini P K and Chapman S J 2007 Mathematical modeling of cell population dynamics in the colonic crypt and in colorectal cancer Proc. Natl Acad. Sci. 1044008

[17] Loeffler M, Stein R, Wichmann H E, Potten C S, Kaur P and Chwalinski S 1986 Intestinal cell proliferation: I. A comprehensive model of steady-state proliferation in the crypt Cell Prolif. 19 627-45

[18] Loeffler M, Potten C S, Paulus U, Glatzer J and Chwalinski S 1988 Intestinal crypt proliferation: II. Computer modelling of mitotic index data provides further evidence for lateral and vertical cell migration in the absence of mitotic activity Cell Prolif. 21 247-58

[19] Lushnikov P M, Chen N and Alber M 2008 Macroscopic dynamics of biological cells interacting via chemotaxis and direct contact Phys. Rev. E 7861904

[20] McDonald S A C, Preston S L, Greaves L C, Leedham S J, Lovell M A, Jankowski J A Z, Turnbull D M and Wright N A 2006 Clonal expansion in the human gut: mitochondrial DNA mutations show us the way Cell Cycle 5 808-11

[21] Meineke F, Potten C S and Loeffler M 2001 Cell migration and organization in the intestinal crypt using a lattice-free model Cell Prolif. 34 253-66

[22] Michor F, Iwasa Y, Rajagopalan H, Lengauer C and Nowak M A 2004 Linear model of colon cancer initiation Cell Cycle $3358-62$

[23] Murray P J 2008 From discrete to continuum models of tumour growth $P h D$ Thesis Oxford University

[24] Murray P J, Edwards C M, Tindall M J and Maini P K 2009 From a discrete to a continuum model of cell dynamics in one dimension Phys. Rev. E 80031912

[25] Murray P J, Kang J W, Mirams G R, Shin S Y, Byrne H M, Maini P K and Cho K H 2010 Modelling spatially regulated $\beta$-catenin dynamics and invasion in intestinal crypts Biophys. J. 99 716-25

[26] Newman T J 2005 Modelling multicellular systems using subcellular elements Math. Biosci. Eng. 2 613-24

[27] Nowak M A, Komarova N L, Sengupta A, Jallepalli P V, Shih I M, Vogelstein B and Lengauer C 2002 The role of chromosomal instability in tumor initiation Proc. Natl Acad. Sci. 99 16226-31

[28] Okamoto R and Watanabe M 2004 Molecular and clinical basis for the regeneration of human gastrointestinal epithelia J. Gastroenterol. 39 1-6

[29] Pitt-Francis J, Bernabeu M O, Cooper J, Garny A, Momtahan L, Osborne J, Pathmanathan P, Rodriguez B, Whiteley J P and Gavaghan D J 2008 Chaste: using agile programming techniques to develop computational biology software Phil. Trans. A 3663111

[30] Pitt-Francis J et al 2009 Chaste: a test-driven approach to software development for biological modelling Comput. Phys. Commun. $1802452-71$

[31] Potten C S and Loeffler M 1990 Stem cells: attributes, cycles, spirals, pitfalls and uncertainties: lessons for and from the crypt Development 110 1001-20

[32] Preston S L et al 2003 Bottom-up histogenesis of colorectal adenomas Cancer Res. 63 3819-25

[33] Reya T and Clevers H 2005 Wnt signalling in stem cells and cancer Nature 434 843-50

[34] Sieber O M, Heinimann K and Tomlinson I P 2003 Genomic instability-the engine of tumorigenesis? Nat. Rev. Cancer $3701-8$

[35] Taylor R W et al 2003 Mitochondrial DNA mutations in human colonic crypt stem cells J. Clin. Invest. 112 1351-60

[36] Van Leeuwen I M M, Byrne H M, Jensen O E and King J R 2006 Crypt dynamics and colorectal cancer: advances in mathematical modelling Cell Prolif. 39 157-81

[37] Van Leeuwen I M M et al 2009 An integrative computational model for intestinal tissue renewal Cell Prolif. 42 617-36

[38] Walter A 2009 A comparison of continuum and cell-based models of colorectal cancer $P h D$ Thesis Nottingham University 